\title{
CAUSE-EFFECT RELATIONS BASED DYNAMIC MODELING AND ITS APPLICATION TO CONTROL*
}

\author{
GANCHO VACHKOV ${ }^{\dagger}$ AND TOSHIO FUKUDA
}

\begin{abstract}
In this paper a special incremental type of cause-effect dynamic model is proposed for use in different control schemes. The model parameters are integrated into the shape of the specially introduced cause-effect relation function. This function represents the degrees of relationship between the past time changes of the control input and the change of the current plant output. The model of the plant dynamics is identified from experimental data by three different algorithms. The first one is a Direct Identification by use of the Least Mean Squares (LMS) algorithm. The second one is the Indirect (reduced size) Identification, which identifies the parameters of an one-dimensional Takagi-Sugeno fuzzy model that is further used to approximate the cause-effect relation function of the dynamic model. Thus a significant reduction in the size of the identification problem is achieved. The third algorithm is called "Soft Guided" Identification that is able to use preliminary human knowledge about the possible or expected type of the plant dynamics. It is able to produce a more plausible dynamic model especially in the presence of highly noised input-output data.

Several versions of predictive control schemes based on the proposed dynamic model are described and investigated in the paper. They use different horizon lengths and horizon widths. Finally, a special version of a feed-forward reference model control, based on the proposed type of incremental cause-effect relation model is described and analyzed in the paper by numerical simulations. All the simulation results are a kind of numerical proof for the real applicability of the proposed dynamic modeling method and the respective control schemes.
\end{abstract}

1. Introduction. Many different approaches to the simulation and identification of dynamic systems have been reported in the literature, some of them using neural networks $[1,2]$, fuzzy models $[3,6]$ or auto regressive techniques $[4,5,6]$ for representing the system behavior and its internal cause-effect relationships. Typical applications of the identified dynamic models are in control, especially predictive type of control $[4,5,7]$ or reference model control, where a precise model with a sufficient degree of generalization ability is of crucial importance.

Let us suppose that a model of a dynamic process $y=y(u, t)$ with an output $y$ and one control input $u$ has to be created by using $M$ discrete time instances $k=1,2, \ldots, M$ with sampling intervals $d t$.

The Nonlinear AutoRegressive with eXogenous input (NARX) model, as in [4, $6]$ is a popular and widely used dynamic model structure, especially for a predictive control purpose. In its common format it defines the non-linear relations between the past inputs, past outputs and the predicted process output, as follows:

$$
\begin{aligned}
y(k+1)= & F\left\{y(k), y(k-1), \ldots, y\left(k-n_{y}+1\right) ;\right. \\
& \left.u\left(k-n_{d}\right), \ldots, u\left(k-n_{u}-n_{d}+1\right)\right\}
\end{aligned}
$$

\footnotetext{
*Invited paper. Received on December 15, 2001; accepted for publication on January 7, 2002.

$\dagger$ Dept. of Micro System Engineering, Nagoya University, Furo-cho, Chikusa-ku, Nagoya 464-8603, JAPAN, Email: vachkov@mein.nagoya-u.ac.jp

${ }^{\ddagger}$ Center for Cooperative Research in Advanced Science and Technology, Nagoya University Furocho, Chikusa-ku, Nagoya 464-8603, JAPAN, E-mail: fukuda@mein.nagoya-u.ac.jp
} 
Here $n_{y}$ and $n_{u}$ are the maximum considered lags for the output and control, respectively and $n_{d}$ is the discrete dead time. The linearized form of the NARX model (with $n_{d}=0$ ) is written in the form:

$$
y(k+1)=\sum_{i=1}^{n_{y}} a_{i} y(k-i+1)+\sum_{i=1}^{n_{u}} b_{i} u(k-i+1)
$$

In case a nonlinear dynamic behavior should be identified, the Takagi-Sugeno (TS) fuzzy model representation $[6,8,9]$ of the $N A R X$ dynamic model can be successfully used. The overall TS fuzzy model interpolates between $L$ local linear ARX models by using the following fuzzy rule base [6]:

$$
\begin{aligned}
& R^{i}: \operatorname{IF}\left\{z_{1}(k) \text { is } A_{1}^{i} \text { and } \ldots \text { and } z_{n}(k) \text { is } A_{n}^{i}\right\}, \text { THEN } y^{i}(k+1)= \\
& c_{0}^{i}+\sum_{j=1}^{n_{y}} a^{i} y(k-j+1)+\sum_{j=1}^{n_{u}} b^{i} u\left(k-j-n_{d}+1\right), \quad i=1,2, \ldots, L
\end{aligned}
$$

The Fuzzy dynamic model constructed in this way has $n$ inputs: $z_{1}(k), \ldots, z_{n}(k)$ $\left(n \leq n_{u}+n_{y}\right)$ and $A_{1}^{i}, A_{2}^{i}, \ldots, A_{n}^{i}$ are the fuzzy sets associated to the $i$-th fuzzy rule $R^{i}$. All fuzzy sets are defined over the following universe of discourse: $\{y(k), \ldots, y(k-$ $\left.\left.n_{y}+1\right) ; u\left(k-n_{d}\right), \ldots, u\left(k-n_{u}-n_{d}+1\right)\right\}$ with dimension $n_{y}+n_{u}$. From a modeling viewpoint, the overall nonlinear dynamic model in (1.3) consists of $L$ linear model cells $M_{i}, i=1,2, \ldots, L$ from the type of (1.2) with nonlinear interconnections and weights that are given by the preliminary tuned TS fuzzy model.

It is obvious that with such a model structure, the accuracy of the overall dynamic model highly depends on the preliminary assumed number of inputs for each model cell (i.e. the number of measured past time samplings for $u(k)$ and $y(k)$ ). However it is also well known $[6,9,11]$ that the fuzzy inference procedure for a fuzzy model with large number of inputs becomes a serious (or even unaffordable) time consuming task since a large number of fuzzy rules should be processed. Therefore a kind of reasonable simplification of the structure of the fuzzy dynamic model by decreasing the number of inputs could be of real practical use. Such incremental type of model for each linear model cell $M_{i}$ that is based on Cause-Effect relations is discussed in the next Section 2 Further on, Sections 3 and 4 of this paper deal with different identification algorithms of the proposed dynamic model. The usage of this dynamic model in different predictive control schemes is given in Sections 5,6 and 7 and application to a feed-forward type of reference model control is given in Section 8. Finally, Section 9 concludes the paper with a brief analysis of the results and future problems.

2. The Incremental Cause-Effect Relation Based Dynamic Model. The dynamics of a plant with control input $u(t)$ and plant output $y(t)$ can be described in an incremental way and discrete form as in $[4,10]$ by use of a cause-effect relations between the change-of-the-past-inputs $\Delta u(k-1), \Delta u(k-2), \ldots$ and the respective change-of-the-current-output $\Delta y(k)$. Further on $n$ denotes the memory length of the 
model and $k$ is the current discrete sampling time. Then the proposed incremental model has the following form:

$$
y(k)=y(k-1)+\Delta y(k)
$$

where:

$$
\begin{gathered}
\Delta y(k)=\varphi[\Delta u(k-1), \ldots, \Delta u(k-i), \ldots, \Delta u(k-n)], \text { with } \\
\Delta u(k-i)=u(k-i)-u(k-i-1)
\end{gathered}
$$

Here, as in [10],we propose and use the following type of cause-effect relation for the function (2.2):

$$
\Delta y(k)=g \sum_{i=1}^{n} \Delta u(k-i) f(i)
$$

that leads to the following incremental dynamic model:

$$
y(k)=y(k-1)+g \sum_{i=1}^{n} \Delta u(k-i) f(i)
$$

Here $g$ is a kind of scaling factor and $f(i), i=1,2, \ldots, n$ are discrete points (parameters) of the so called Cause-Effect-Relation Function (CER-functions). Each point $f(i)$ of this function represents the strength degree of the relationship between the past change of the control $\Delta u(k-i)$ and the resulted change of the output $\Delta y(k)$ at the current $k$-th sampling time.

The above proposed dynamic model (2.5) will be further referred to as Incremental Cause-Effect Relation (ICER) dynamic model.

Figure 1 shows an example of five ICER models with different CER-functions, representing five different dynamics as shown in Fig. 2.

It should be noted that the scaling factor $g$ is used just as a normalizing coefficient that allows different CER-functions to be compared by shape. It does not have a meaning of gain $G$ of the model, as in the classic control theory. That gain is implicitly included into the shape and the length of the CER-function and can be easily calculated by:

$$
G=\frac{\Delta y}{\Delta u}=g \sum_{i-1}^{n} f(i)
$$

It is often the case when the process gain $G$ of the current CER-function $f(i)$ should be changed to a desired (reference) value $G_{r}$ while keeping the same type of dynamics 


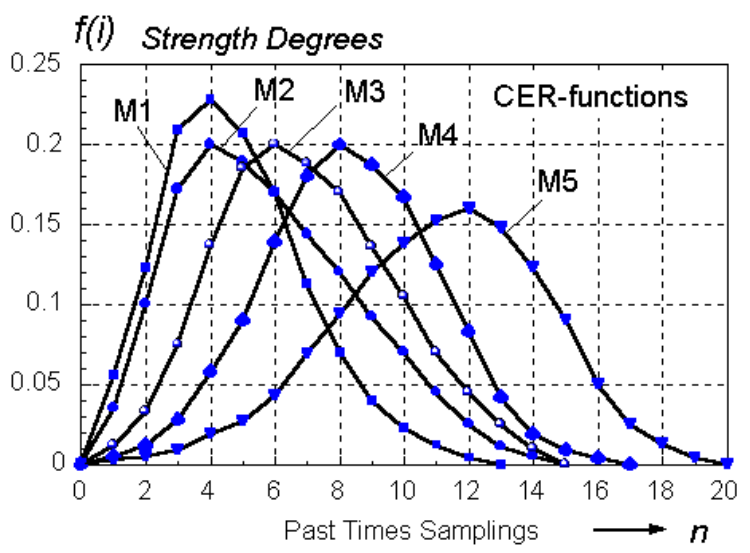

FIG. 1. Five ICER Dynamic Models with Different Cause-Effect Relation Functions

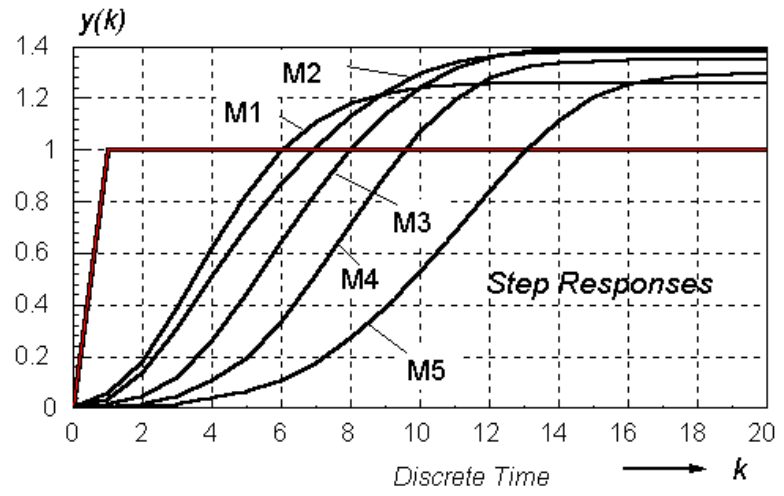

FIG. 2. Step Responses of the five ICER Dynamic Models from Fig. 1.

as represented by the shape of the CER-function $f(i)$. Then taking into account (2.6), the new (reference) function $f_{r}(i)$ will be calculated as follows:

$$
f_{r}(i)=\frac{G_{r}}{G} f(i), \quad i=1,2, \ldots, n
$$

The shape of the above introduced CER-function carries certain information about the type of dynamics of the plant. By changing this shape different (practically all) types and orders of dynamics can be represented, including also time delay dynamic processes. This makes the proposed structure of the dynamic model a convenient tool for describing different types of dynamics. Additionally, the clear physical meaning of the CER-function gives a possibility for utilizing a preliminary and subjective human knowledge in the identification process.

For some dynamic processes the CER-function $f(i)$ is known in advance or could be defined approximately by a human expert and plant operator, based on preliminary knowledge about the process. In other cases no preliminary information about the process exists, so that plant dynamics should be identified based on the available 
experimental input-output process data.

It can be easily noticed that the above proposed incremental cause-effect (ICER) model in (2.5) is equivalent to the following class of simplified linearized NARX model:

$$
y(k)=a_{1} y(k-1)+\sum_{i=1}^{n_{u}} b_{i} u(k-i)
$$

Equations describing the transition from the given ICER model in (2.5) to its equivalent NARX model in (1.2) are written as follows:

$$
\begin{aligned}
& a_{1}=1 ; b_{1}=f(1) ; \\
& b_{i}=f(i)-f(i-1), i=2, \ldots, n ; \\
& n_{u}=n+1 ; \quad b_{n_{u}}=b_{n+1}=-f(n) .
\end{aligned}
$$

The above equations could be useful, for example, if there is a need to perform a predictive control algorithm using the NARX model, but the identification of the process has been performed as an ICER model due to existence of a human knowledge about the process dynamics.

3. Direct Identification of the Dynamic Model Based on the Least Mean Squares Algorithm. This is an off-line identification problem where all $n$ strength degrees $f(i), i=1,2, \ldots, n$ of the CER-function have to be identified based on experimental data. From an optimization point of view the mean squared error (MSE) should be minimized, in the form of the following criterion:

$$
Q=\frac{1}{m} \sum_{k=n+1}^{M}\left\{d(k)-\left[y(k-1)+\sum_{i=1}^{n} \Delta u(k-i) f(i)\right]\right\}^{2}
$$

where $d(k)$ denotes the measured output from the real process and $m=M-n$ is the real amount of experimental data used in the identification. This problem can be solved by the direct implementation of the Least Mean Squares (LMS) algorithm, as shown in [10].

The basic linear system of equations is rectangular in size, as follows:

$$
\text { Af }=\mathbf{d}
$$

The $m$ sized vector $\mathbf{d}$ has the following structure:

$$
\mathbf{d}=[\Delta y(n+1), \Delta y(n+2), \ldots, \Delta y(M)]^{T}
$$

The unknown vector $\mathbf{f}$ of the $C E R$-function strength degrees with size $n$ has a format:

$$
\mathbf{f}=\left[\begin{array}{lllll}
f(1) & f(2) \ldots f(i) \ldots f(n)
\end{array}\right]^{T}
$$

The rectangular matrix $\mathbf{A}$ with size $m \times n$ is constructed as follows:

$$
\mathbf{A}=g\left[\begin{array}{cccc}
\Delta u_{n+1}(k-1) & \Delta u_{n+1}(k-2) & \cdots & \Delta u_{n+1}(k-n) \\
\Delta u_{n+2}(k-1) & \Delta u_{n+2}(k-2) & \cdots & \Delta u_{n+2}(k-n) \\
\cdots & \cdots & \cdots & \cdots \\
\Delta u_{M}(k-1) & \Delta u_{M}(k-2) & \cdots & \Delta u_{M}(k-n)
\end{array}\right]
$$


Then the original rectangular linear system (3.1) is modified into a square system of equations with size $n$ :

$$
\mathbf{A}^{\mathbf{T}} \mathbf{A} \mathbf{f}=\mathbf{A}^{\mathbf{T}} \mathbf{d}
$$

Finally, the solution of the above identification problem is represented in a LeastMean-Square sense as:

$$
\mathbf{f}=\left(\mathbf{A}^{\mathbf{T}} \mathbf{A}\right)^{-\mathbf{1}} \mathbf{A}^{\mathbf{T}} \mathbf{d}
$$

This standard Identification scheme will be further referred to as Direct Identification of the ICER model.

4. Reduced Size Indirect Identification Based on Takagi-Sugeno Fuzzy Model Tuning. It is well known that fuzzy models, especially Takagi-Sugeno (TS) fuzzy models $[8,9,11,6]$, are widely used as universal approximators of nonlinear relationships. This fact suggests the fruitful idea to utilize the TS model in order to reduce the dimensionality and therefore the computational cost of the original stated Identification problem in the previous section. The newly proposed method is further called Indirect Identification of the CER-function [10]. It is a kind of modification of the Direct Identification (by use of the standard LMS algorithm) where all the parameters of the CER-function are implicitly identified by only identifying the consequent parameters of a one-dimensional TS fuzzy model. That TS fuzzy model serves later as an approximator to retrieve all points of the CER-function.

The obvious computational advantage of this Indirect Identification approach is the reduction in the size of the original Identification problem. Indeed the number of the consequent parameters of the one-dimensional TS fuzzy model is much less (usually no more than 8-10 parameters) than the number $n$ of the points in the CERfunction $f(i)$ being 20,30 or even more for slow and complex dynamic processes. Further on, the details of this algorithm are given.

Suppose that the cause-effect relation function $f(i), i=1,2, \ldots, n$ has to be approximated by a one-dimensional TS fuzzy model with $L$ Gaussian membership functions and linear consequent parts of the fuzzy rules. Then the number of the fuzzy rules coincides with the number $L$ of the membership functions. If the centers and widths of each membership function are preliminary fixed, then the identification problem of the fuzzy model is transformed into finding all $2 L$ consequent parameters of the model. The later task is solved by a modification of the LMS algorithm, that adjusts the linear consequent parameters $p_{0 r}$ and $p_{1 r}, r=1,2, \ldots, L$ of the fuzzy model, so that to fit as close as possible all $n$ points $f(i), i=1,2, \ldots, n$ of the CER-function. The details are given below.

Since each point of the CER-function has to be matched by the fuzzy model, the following equations hold:

$$
f(i)=\sum_{r=1}^{L} \nu_{r}(i)\left(p_{r 0}+p_{r 1} i\right), i=1,2, \ldots, n
$$


Here $p_{r 0}$ and $p_{r 1}$ are the linear consequent parameters of the $r$-th fuzzy rule $(r=$ $1,2, \ldots, L)$ and $\nu_{r}(i)$ is the normalized truth value (activation degree) of the $r$-th fuzzy rule for the $i$-th approximation point

$$
\nu_{r}(i)=\mu_{r}(i) / \sum_{j=1}^{L} \mu_{j}(i), i=1,2, \ldots, n
$$

The fuzzified value $\mu_{r}(i)$ for the $i$-th point by the $r$-th Gaussian membership function $(r=1,2, \ldots, L)$ is calculated as:

$$
\mu_{r}(i)=\exp \left(-\frac{\left(i-c_{r}\right)^{2}}{2 \sigma_{r}^{2}}\right), i=1,2, \ldots, n
$$

Here $c_{r}, \sigma_{r}, r=1,2, \ldots, L$ are the predetermined centers and widths of the respective membership functions. Note that the one-dimensional fuzzy model stated in this way will be used as approximator of the CER-function thus and will have only discrete numbers $i,(i=1,2, \ldots, n)$ in its input.

By substituting $f(i)$ in (2.4) with its expression from (4.1), the following rectangular system of equations with size $m \times 2 L$ is obtained:

$$
\begin{array}{r}
\Delta y(k)=g \sum_{i=1}^{n} \Delta u(k-i) \sum_{\substack{r=1 \\
k=n+1, n+2, \ldots, M}}^{L} \nu_{r}(i)\left(p_{r 0}+p_{r 1} . i\right), \\
k=n+n
\end{array}
$$

The LMS solution of the above system for obtaining the consequent parameters $p_{0 r}$ and $p_{1 r}, r=1,2, \ldots, L$ is given in [10].

For illustration, in Fig. 3 and Fig. 4. an example of such Indirect Identification is given for the case of TS fuzzy model with four Gaussian membership functions $(L=4)$ and four different memory lengths of the CER-functions, as follows: $n=$ 25, 20, 15, 12. The bold line in Fig. 4. denotes the original CER-function (with $n=30)$ that has to be identified.

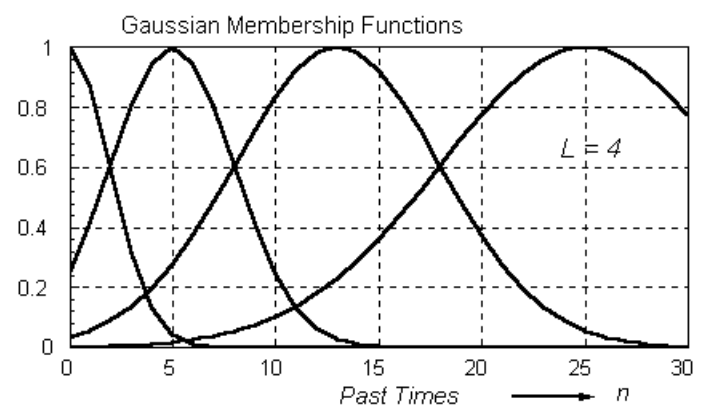

FIG. 3. Four Predetermined Gaussian Membership Functions Used for the Indirect Identification. 


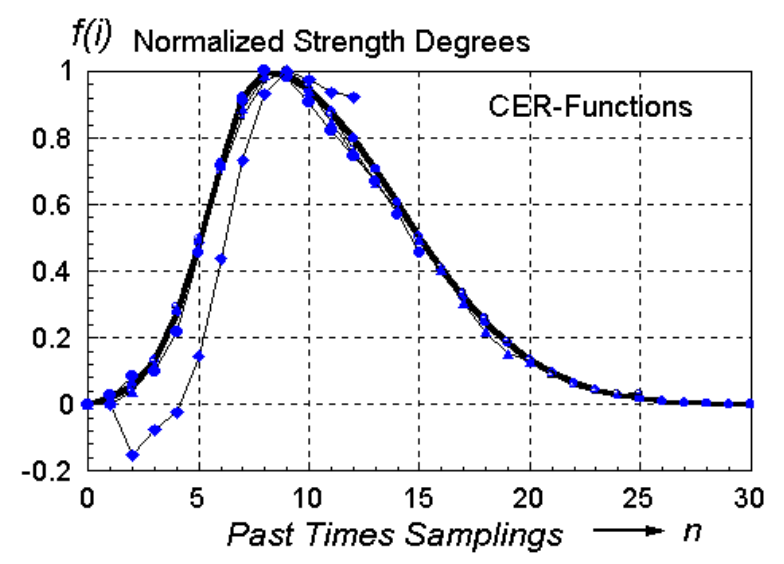

FIG. 4. Indirect Identification of four CER-functions with different memory lengths $n=$ $25,20,15,12$.

For the purpose of identification, an input signal with 80 samplings has been applied to the original ICER model and the respective inputs-output pairs of data have been used to construct the linear system in (4.4). As seen in Fig. 4., the indirect identification with $n=12$ fails to find the shape of the original CER-function, because of a big loss in information (too short memory). The other identifications (with memory legths $n=15,20,25$ ) are satisfactory and give results that are very closed to the real shape of the original CER-function.

It should be noted that in real applications, the actual memory length $n$ of the CER-function is unknown and should be predicted. One way to do this is to examine the shapes of several identified CER-functions and analyse their plausibility and physical meaning, when possible.

5. "Soft-Guided" Identification Algorithm. From a mathematical point of view, the Identification schemes in the above Sections 3 and 4 can be characterized as pure data fitting algorithms. It means that they try to only fit the experimental data according to a pre-specified criterion such as (3.1) (minimizing the MSE), without "taking care" of the physical meaning of the model. Therefore if the data set is highly noisy and/or includes effects of unmeasured inputs with non-zero means, then the identified model may largely deviate from the real process dynamics, thus losing its physical meaning. In such cases an utilization of any kind of preliminary or subjective human knowledge about the type of the model and/or approximate process dynamics could be helpful for the final accuracy and plausibility of the identification process, preventing it from going into an "undesirable" direction.

The proposed above ICER model is able to implement some kind of human knowledge about the dynamics of the process by suggesting the approximate shape of the membership function $f(i)$. However the algorithms in Sections 3 and 4 cannot utilize such information. 
The following numerical example explains graphically some of the demerits of the Direct Identification schemes in Sections 3 and 4. In Fig. 5. a test input-output data set without noise is generated by using the ICER model $M 3$ from Fig. 1.

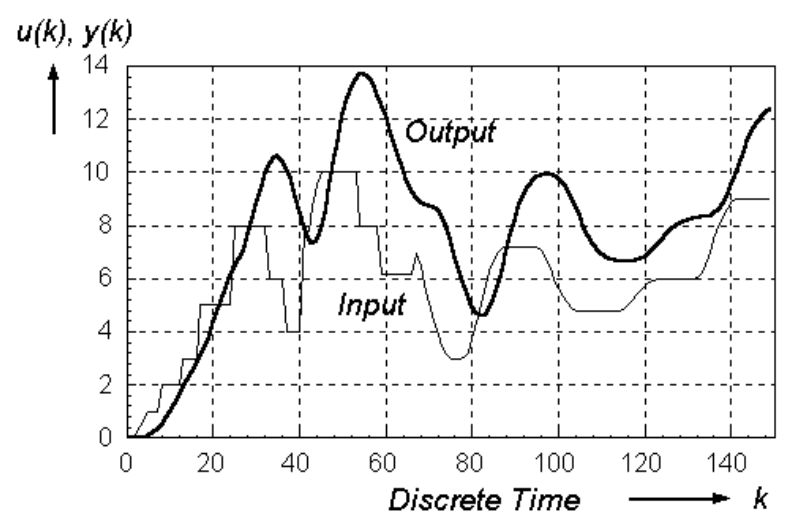

FIG. 5. Pure Input-Output Data Set used for the Direct Identification of the ICER Model M3 from Fig. 1.

The Direct Identification scheme (3.7) finds exactly the model. After that the same dynamic process has been "contaminated" by additive noise with uniform distribution within the interval $[-2,+2]$. The obtained noised input-output data set has been used further for identification and the results are depicted in Fig. 6.

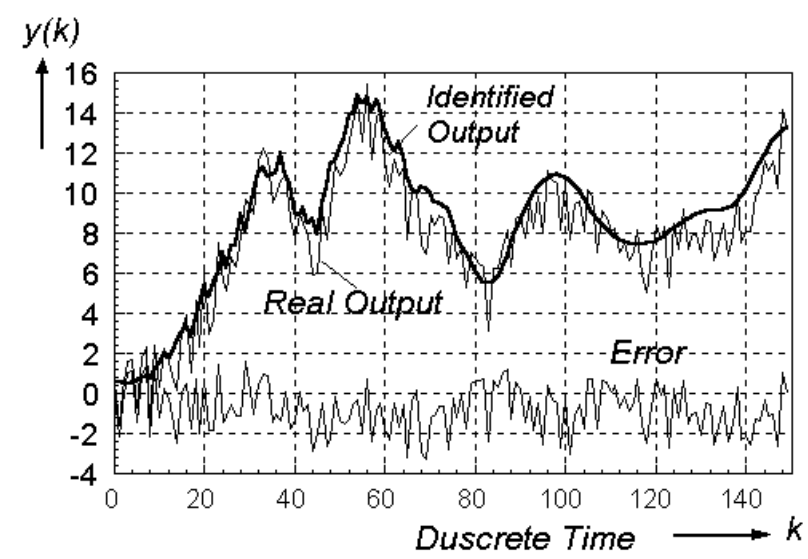

FIG. 6. Noised Input-Output Data Set used for the Direct Identification of the ICER Model M3.

The identified ICER model is shown in Fig. 7. and compared with the original CER-function of the model $M 3$ from Fig. 1. It is obvious that the identification fails to discover the essential input-output relationships of the process. The calculated output by use of the poorly identified ICER model from Fig. 7. is shown by bold line 
in Fig. 6.

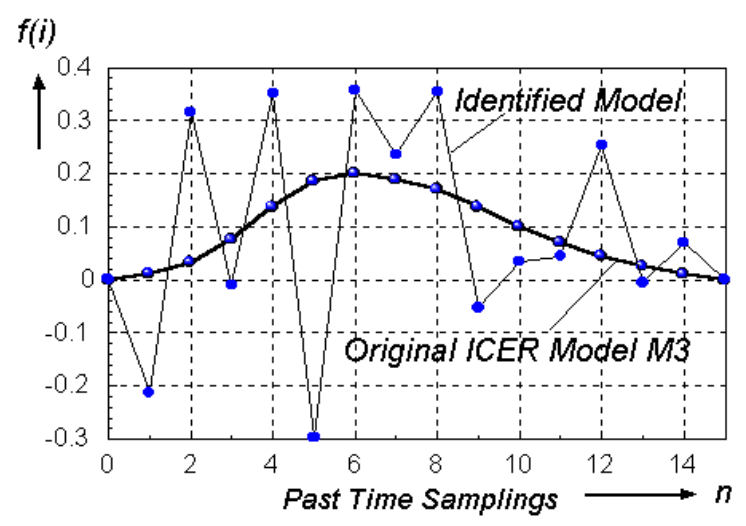

FIG. 7. The Identified CER Model from the Noisy Data in Fig. 6.

Taking into account the above disadvantages, in the sequel another identification algorithm is described, based on a kind of hybrid criterion that includes the human knowledge about the identified process.

This algorithm is further called "Soft-Guided" Identification and tries to minimize the following criterion:

$$
Q=\sum_{k=n+1}^{M}[d(k)-y(k)]^{2}+\sum_{i=1}^{n} \lambda(i)\left[f_{r}(i)-f(i)\right]^{2}
$$

where $f_{r}(i)$ represents the human knowledge (suggestion) about the most plausible or desirable membership function of the process. The selection of the weighting coefficients $\lambda(i), i=1,2, \ldots, n$ is important for the proper balance in the identification process between the pure data fitting (if $\lambda(i) \rightarrow 0$ ) and the pure membership function fitting (if $\lambda \rightarrow \infty$ ).

The two sums in the criterion (5.1) have different nature and different number of elements. Therefore we propose here a kind of normalization scheme with equal weighting coefficients for all $n$ points, that is:

$$
\lambda(1)=\lambda(2)=\ldots=\lambda(n)=\lambda
$$

where

$$
\lambda=\alpha\left(R_{\max }-R_{\min }\right) / f_{r \max }
$$

with $\left[R_{\min }, R_{\min }\right]$ being the output range of the measured data $d(k)$ and $f_{r \max }$ as the peak of the human specified (reference) membership function. In this way the identification can be performed with a human defined factor $\alpha$ within the range $\alpha \in$ [0, 100]. 
The final solution of the identification problem (5.1) is represented again in a LMS sense by (3.7) with an appropriate modification of the matrices (3.3) and (3.5). However this time the result of the identification takes into consideration the existing human knowledge or the human suggestion about the dynamics type, as implemented in (5.1).

The following numerical simulations demonstrate the effect of using the proposed "Soft-Guided" Identification in the case of the highly noisy process from Fig. 6.

We consider the case when the human operator has a kind of preliminary knowledge or perception that the process dynamics should be similar to that one of the model $M 2$ in Fig. 1. even if the true dynamics is actually M3. Then Fig. 8. shows the results from five different "soft-guided" identifications with different factors $\alpha$ as follows: $1,5,10,30,50$. It is seen that the identified models gradually approach the reference model $M 2$ and become smoother in shape, which is more meaningful. The respective unit step responses of some of the identified models are depicted in Fig. 9. for $\alpha=0,1,3,5,10$. They also become gradually closer to the desired reference model $M 2$.

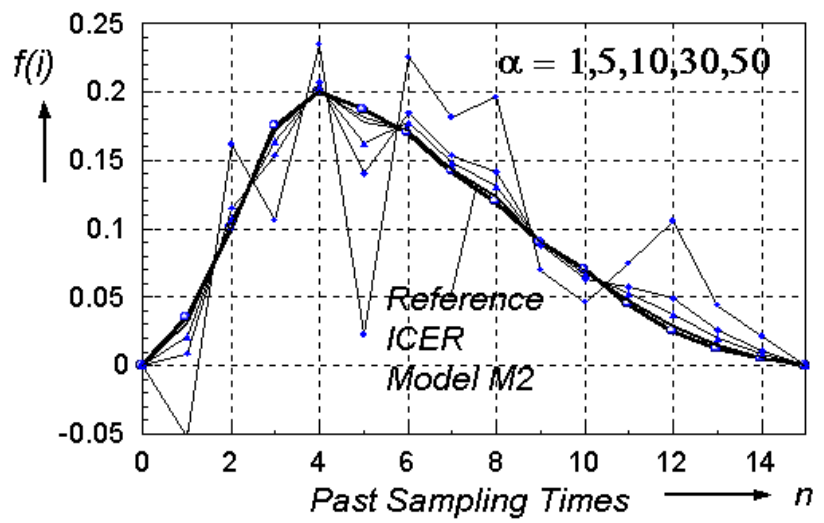

FIG. 8. Identified ICER Models from the Noisy Data in Fig. 6. with Different Factors $\alpha$ and a Reference Model M2.

It is worth to note that the mean square error (MSE) taken over all output data $y(k)$ shows a slight tendency of increasing by increasing $\alpha$. This could be logically explained by the fact that in this case the identified model is gradually forced to follow the reference model $M 2$, which is actually different from the true model $M 3$, as seen in Fig. 1.

If a true reference model $M 3$ is selected as a reference model, the same identification experiments produce an MSE that is monotonically decreasing by increasing $\alpha$. Such a tendency could be practically used as a clear sign that the human knowledge about the reference model approach the true model or at least is close to the real 


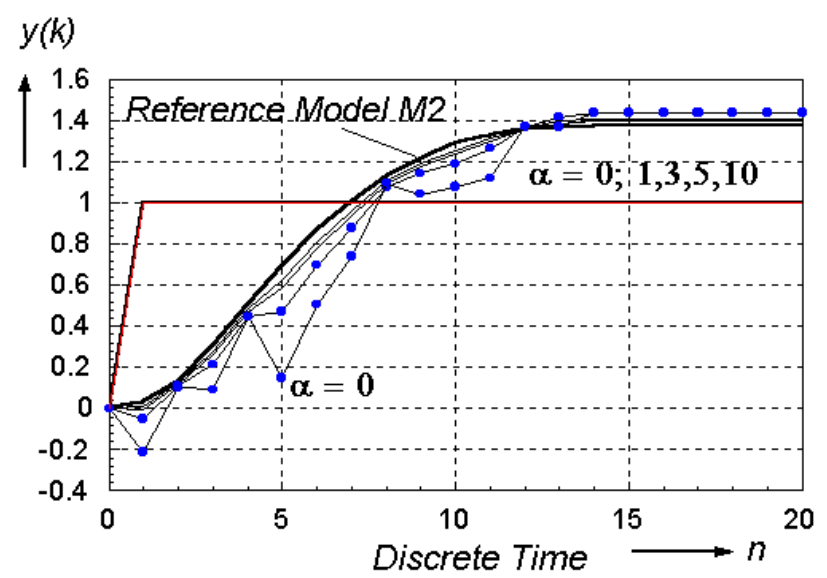

FIG. 9. Unit Transient Processes of Different Identified ICER Models by Use of the Reference Model M2.

process dynamics. Thus the proposed "Soft-Guided" Identification scheme can also serve as a kind of diagnostic tool to discover the most plausible model of the process from a noisy data collection.

6. Simple Predictive Control by Use of the ICER Dynamic Model. Model predictive control $[4,5,7]$ is a powerful tool for dynamic optimization and control of processes with large time delay and time-varying parameters. It is based on a specified (identified) dynamic model of the plant and on-line measurements in order to build a prediction of the future output behavior. On the basis of this prediction, an optimization is performed to find the sequence of future controls that minimize the chosen measure of the model output deviation from a preliminary specified reference trajectory of the plant.

In the simplest case, the aim of the predictive control is to generate such a control $u(k)$ that would produce a model output $y(k+1)$ equal to the reference (desired) value $r(k+1)$ at the future time instant $k+1$, that is: $y(k+1)=r(k+1)$. This scheme is called: one-step-ahead predictive control.

In the more general case of predictive control [4], the notion of receding horizon is defined as a range $\left[h_{1}, h_{2}\right]$ with $h_{2} \geq h_{1}$, a horizon length $h$ given by $h=h_{1} \geq 1$ and a preliminary defined horizon width: $w=h_{2}-h_{1}+1$. Figure 10 . gives an illustration to the general predictive control scheme.

The aim of the general predictive control algorithm is to minimize the deviation of the model output from the given plant reference trajectory within the horizon width $w$ by considering not only one, but rather a series of multiple control actions in the future, namely $u(k+1), u(k+2), \ldots$

If the ICER dynamic model (2.5) is identified by either of the above proposed identification schemes, it can be easily implemented into various predictive control 


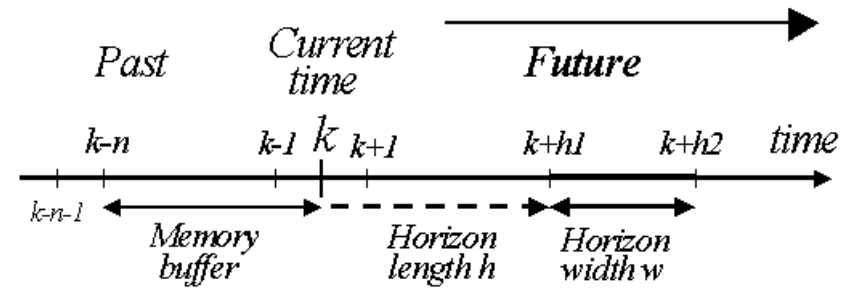

FIG. 10. General Predictive Control Scheme

schemes as in $[4,5,7]$. Let us consider the simplest case of a predictive control with one-step-ahead prediction (horizon length $h=1$ ). The future (next sampling) model output is given as:

$$
y(k+1)=y(k)+\Delta y(k+1)
$$

with:

$$
\begin{aligned}
\Delta y(k+1) & =y(k+1)-y(k)=g \sum_{i=1}^{n} \Delta u(k+1-i) f(i) \\
& =g \Delta u(k) f(1)+g \sum_{i=2}^{n} \Delta u(k+1-i) f(i)
\end{aligned}
$$

After simple algebraic transformations the following equation for computing the required control $u(k)$ at the current sampling time is obtained:

$$
u(k)=u(k-1)+\frac{r(k+1)-y(k)-g \sum_{i=2}^{n} \Delta u(k+1-i) f(i)}{g f(1)}
$$

The control scheme with one-step-ahead prediction usually does not give good performance results $[4,7]$ and often leads to instability and oscillations, while requiring large control actions. The general reason for such performance is that there is a weak relationship between the control $u(k)$ and the result $y(k+1)$ at the next time instant (see $f(1)$ in Fig. 1.), which leads to a weak controllability of the process. This is especially true for processes with larger time delay. As a result the control $u(k)$ is chattering between the predetermined constraints: $u_{\min }$ and $u_{\max }$.

A better and smoother control can be realized with a longer prediction horizon $h>1$.

7. Predictive Control with Longer Prediction Horizon. This is a more general case of a predictive control where $h=h_{1}>1$ and more future control actions could be calculated. Therefore another parameter called length of the control horizon $n_{c} \leq h_{2}$ should be determined beforehand. It represents the number of the different control actions in the future $u(k), u(k+1), \ldots, u\left(k+n_{c}-1\right)$ that have to be calculated in order to optimize the performance of the predictive control within the given horizon range $\left[h_{1}, h_{2}\right]$ with width $w=h_{2}-h_{1}+1$. 
In this paper we consider the simplified case of only one control action $n_{c}=1$. It means that the optimization of the performance within the horizon range $\left[h_{1}, h_{2}\right]$ is carried out by only one control $u(k)$ and after that the projected control increments are assumed to be zero, that is:

$$
u(k+1)=u(k+2)=\ldots=u\left(k+n_{c}\right)=u(k)
$$

The above assumption is an obvious simplification that leads to one-dimensional optimization task. However it does not change the main idea of the predictive control based on the proposed incremental cause-effect relation model (6.1) and (6.2).

There are two possible cases of predictive control with a longer horizon $h=h_{1}>$ 1, differing from the horizon width $w=h_{2}-h_{1}+1$, as follows: $w=1$ and $w>1$. The respective predictive control schemes are discussed in the sequel.

\subsection{Predictive Control with Longer Horizon and One-Point Horizon}

Width. According to the notations in Fig. 5., this case of predictive control can be expressed as:

$$
n_{c}=1 ; h=h_{1}>1 ; h_{2}=h_{1} ; w=1
$$

In order to calculate the control action $u(k)$, the following model recursion is used:

$$
y(k+2)=y(k+1)+\Delta y(k+2)
$$

and further on:

$$
y(k+2)=y(k)+\Delta y(k+1)+\Delta y(k+2)
$$

Then the change of the output is calculated as:

$$
\begin{aligned}
\Delta y(k+2) & =g \sum_{i=1}^{n} \Delta u(k+2-i) f(i) \\
& =g \Delta u(k) f(2)+\sum_{i=3}^{n} \Delta u(k+2-i) f(i)
\end{aligned}
$$

As seen the term containing $\Delta u(k+1)$ is omitted in (7.5), in accordance with the assumption (7.1) to keep the control unchanged in the future samplings. Finally, the predicted output at the end of the horizon with length $h$ will be:

$$
y(k+h)=y(k)+\sum_{j=1}^{h} \Delta y(k+j),
$$

where

$$
\Delta y(k+j)=g \sum_{i=j+1}^{n} \Delta u(k+j-i) f(i)+g \Delta u(k) f(h), j=1,2, \ldots, h
$$


Under the assumption that a perfect control at the future time instant $k+h$ has to be achieved, which is represented as : $y(k+h)=r(k+h)$, the change of the control action at the current time $k$ will be:

$$
\Delta u(k)=\frac{r(k+h)-y(k)-D}{g \sum_{j=1}^{h} f(j)},
$$

where

$$
D=g \sum_{j=1}^{h} \sum_{i=j+1}^{n} \Delta u(k+j-i) f(i)
$$

Note that for any longer horizon: $h>n$, the CER-function becomes zero, namely: $f(h)=0$. Finally, the applied control at the current sampling time $k$ is given by:

$$
u(k)=u(k-1)+\Delta u(k)
$$

7.2. Predictive Control with Longer and Wider Horizon. This is a more general case of the predictive control with the following parameters, according to Fig. $5 .:$

$$
n_{c}=1 ; h=h_{1}>1 ; h_{2}>h_{1} ; w=h_{2}-h_{1}+1>1 ;
$$

Here the control performance should be optimized over the whole horizon range $\left[h_{1}, h_{2}\right]$ with $m$ points as follows: $h_{1}, h_{1}+1, \ldots, h_{2}$ by using only one control $u(k)$. The solution of this problem is a further development of equations (7.8) and (7.9) that leads to a least mean squares solution of a linear system of equations, as shown in [12].

\section{Simulation Results for Predictive Control by Use of the ICER Dy-} namic Model. Extensive simulations have been performed in order to evaluate the merits and demerits of the proposed incremental dynamic model (ICER model) based on cause-effect relation functions model in different predictive control schemes. A hypothetical plant and its identified model have been assumed for the further simulation with CER-function, as shown in Fig. 11.

First the ideal case of perfect identification of the plant has been assumed, that is: Model $=$ Plant in order to study the performance of the different predictive control schemes. A reference trajectory $r(k)$ with arbitrary shape and 140 samplings has been assumed in the simulations. As mentioned in Section 6, the one-step-ahead predictive control $(h=1)$ shows instability because of the time delay and low controllability of the plant.

Simulation results for the predictive control with one point horizon width $(w=1)$ and different horizon lengths $h=2 \leftrightarrow 7$ are shown in Fig. 12 . 


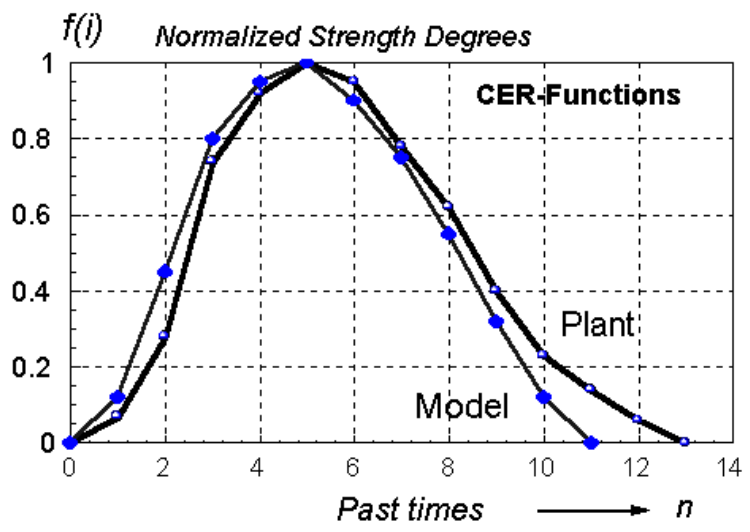

FIG. 11. Assumed Dynamics of the Plant and Model.

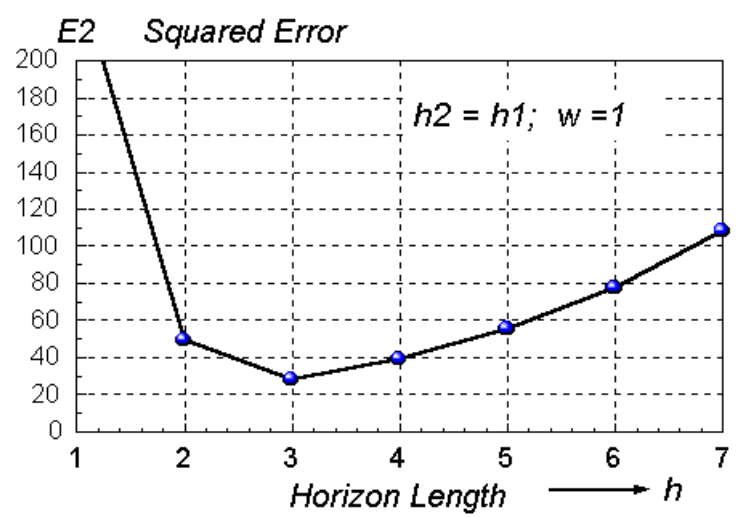

Fig. 12. Predictive Control Performance with Different Horizon Lengths $h$.

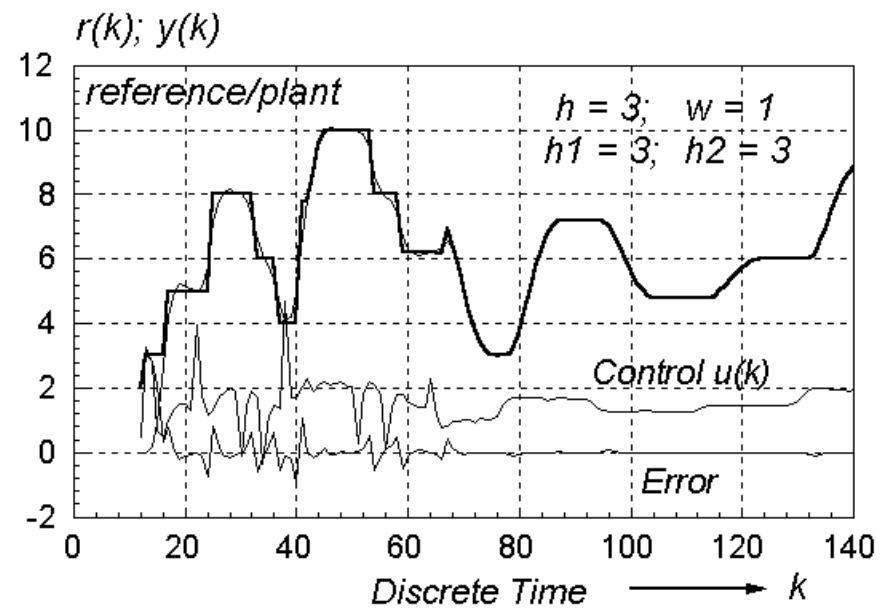

FIG. 13. Predictive Control with Three-Steps-Ahead Prediction $(h=3)$ and Unit Horizon Width $(w=1)$. 
Figure 13. shows the results of the predictive control with horizon width: $w=1$ and a horizon length: $h=3$ under the assumption of exact identification of the plant $($ Plant $=$ Model $)$.

Further simulations have been conducted where a certain difference between the identified model and the plant exists (Model $\neq$ Plant), as shown in Fig. 11., due to the inexact identification. Figure. 14. depicts such a case of a predictive control with horizon length $h=3$ and width $w=1$ by using the inexact identified ICER model. The control performance has been obviously deteriorated which shows the importance of the model accuracy in the predictive control scheme.

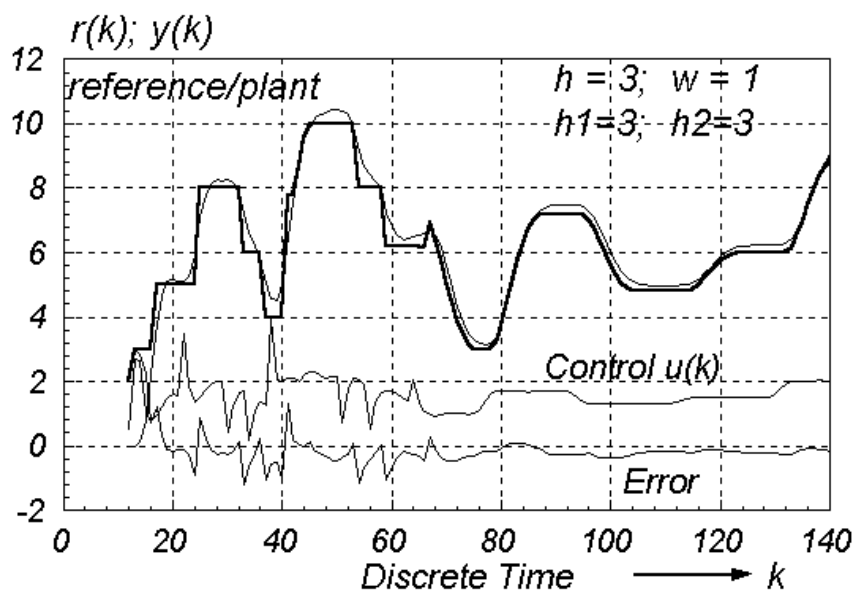

Fig. 14. Predictive Control in the Case of Difference Between the Plant and the Model

\section{Feed Forward Reference Model Control by Use of the ICER Dy-} namic Model. The above described cause-effect relation dynamic model in Section 2 can be also successfully used in other control schemes. One of them with certain application areas in robotics and other engineering fields is the so called reference model control [2]. Here the desired dynamic behavior of the plant is given by a predefined and stable Reference Model. The main goal of the controller is to force the process to follow the reference model output. In the simplest case, the controller could be of the type of feed-forward controller, as shown in Fig. 15.

From control point of view, such a feed-forward controller can be regarded as a special "correction unit" that transforms the initial reference signal $r(t)$ into a modified control $u(t)$ which is further applied as a plant input.

In order to design such a controller (correction unit), an identification procedure based on experimental data should be performed. Let us assume that the dynamics of all units is represented by the proposed ICER dynamic model with CER-functions 


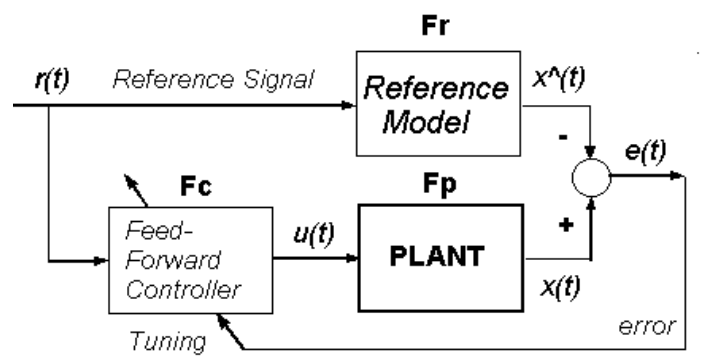

FIG. 15. Reference Model Control Structure with Tuning of the Feed-Forward Controller.

and memory lengths as follows: $F_{r}$ and $n_{r}$ for the Reference Model; $F_{p}$ and $n_{p}$ for the Plant and $F_{c}$ with $n_{c}$ for the Controller. Suppose that $k_{\max }$ sampling times from the reference signal $r(k)$ are also needed for the identification. Then the general statement of this identification problem is as follows:

Given $F_{r}, n_{r} ; F_{p}, n_{p} ; k_{\max }$ and $n_{c}$; determine all $n_{c}$ points of the CER-function $F_{c}$ of the Controller that minimize the following performance index:

$$
Q=\frac{1}{2} \sum_{k=1}^{k_{\max }}[x(k)-\hat{x}(k)]^{2}
$$

The solution of this identification problem can be obtained in a non-iterative way by a proper modification of the LSM algorithm.

Let us first derive the output of the serial connection of the controller (correction unit) and the Plant, as shown in Fig. 16.

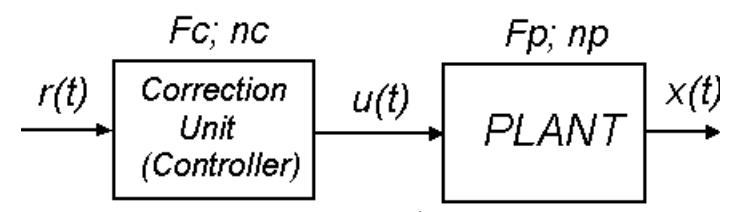

FIG. 16. Serial Connection of two Dynamic Units.

$$
\begin{gathered}
x(k)=x(k-1)+\sum_{i=1}^{n_{p}} \Delta u(k-i) f_{p}(i) \\
\Delta u(k-i)=u(k-i)-u(k-i-1)=\sum_{j=1}^{n_{c}} \Delta r(k-i-j) f_{c}(j)
\end{gathered}
$$

Finally the output of the plant is calculated as:

$$
x(k)=x(k-1)+\sum_{i=1}^{n_{p}} f_{p}(i) \sum_{j=1}^{n_{c}} \Delta r(k-i-j) f_{c}(j)
$$


Obviously, the total memory length $n$ for the whole serial connection unit, which represents the number of all past times used for calculation of the current output $x(k)$ is: $p=n_{p}+n_{c}$.

In the identification scheme, according to Fig. 15., we assume that the increments of the reference model and that one of the serial connection of the controller and the plant, are equal at each sampling time $k$, that is:

$$
\Delta \hat{x}(k)=\Delta x(k)
$$

which yields:

$$
\begin{aligned}
x(k)=\sum_{j=1}^{n_{p}} \Delta u(k-j) f_{p}(j) & =\sum_{j=1}^{n_{p}} f_{p}(j) \sum_{l=1}^{n_{c}} \Delta r(k-j-l) f_{c} \quad \text { and } \\
\sum_{i=1}^{n_{r}} \Delta r(k-i) f_{r}(i) & =\sum_{j=1}^{n_{p}} \Delta u(k-j) f_{p}(j) \\
& =\sum_{j=1}^{n_{p}} f_{p}(j) \sum_{l=1}^{n_{c}} \Delta r(k-j-l) f_{c}
\end{aligned}
$$

Finally, the solution $f_{c}(l), l=1,2, \ldots, n_{c}$ for the correction unit is obtained by solving the following linear system of equations:

$$
A_{l}(k) . f_{c}(l)=B(k), \quad l=1,2, \ldots, n_{c} ; k=1,2, \ldots, k_{\max } ; k_{\max }>n_{c}
$$

where:

$$
\begin{gathered}
A_{l}(k)=\sum_{j=1}^{n_{p}} f_{p}(j) \Delta r(k-j-l) \quad \text { and } \\
B(k)=\sum_{i=1}^{n_{r}} \Delta r(k-i) f_{r}(i)
\end{gathered}
$$

The above Identification scheme (9.9), (9.10) and (9.11), has been used for extensive simulations of the Reference Model control by use of the ICER Dynamic Model, in accordance with the control structure in Fig.15. The assumed plant and reference model dynamics are shown in Fig. 17. A portion of 120 samplings from a reference signal with stochastic behavior has been used for the identification. 


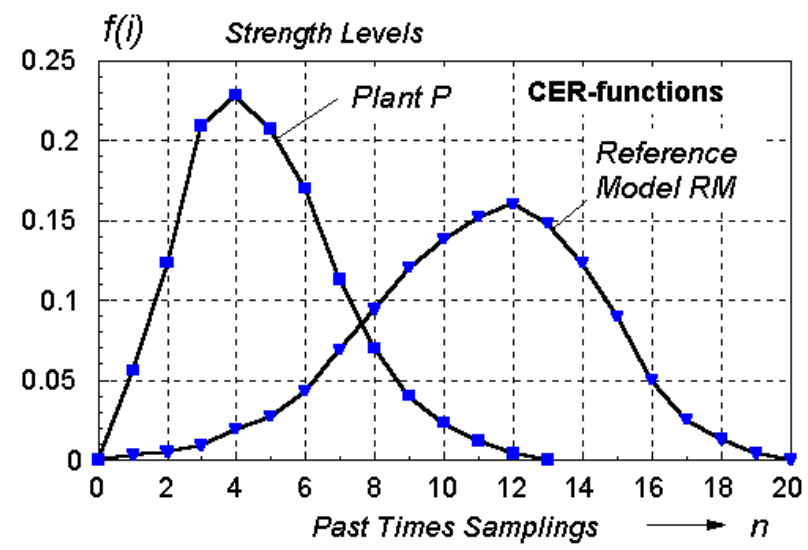

FiG. 17. Dynamics of the Plant and the Reference Model.

Two groups of identified feed-forward controllers are shown in Fig. 18. and Fig. 19. with different memory lengths within the range $n_{c} \in[2,12]$. As seen from these figures, the CER-functions for the controllers are represented by (seems to be) broken lines in contrast with the smooth-shaped CER-functions for the plant and the reference model from Fig. 17. Therefore these CER-functions cannot be used for direct extraction of a physical meaning that explains the behavior of the controllers.

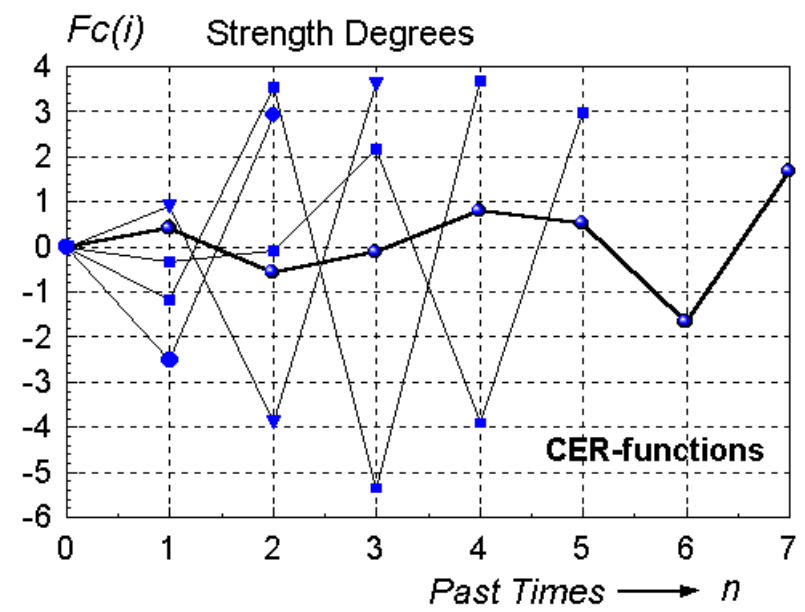

FIG. 18. Identified CER-functions of the Feed-Forward Controllers with Different Memory Lengths: $n=2,3,4,5$ and 7 .

Because of the difference between the memory lengths of the plant and because the reference model is $7\left(n_{p}=12\right.$ and $\left.n_{r}=19\right)$, it is expected that a controller with a memory length $n_{c}=7$ would perform well. The following Fig. 20. and Fig. 21. serve 


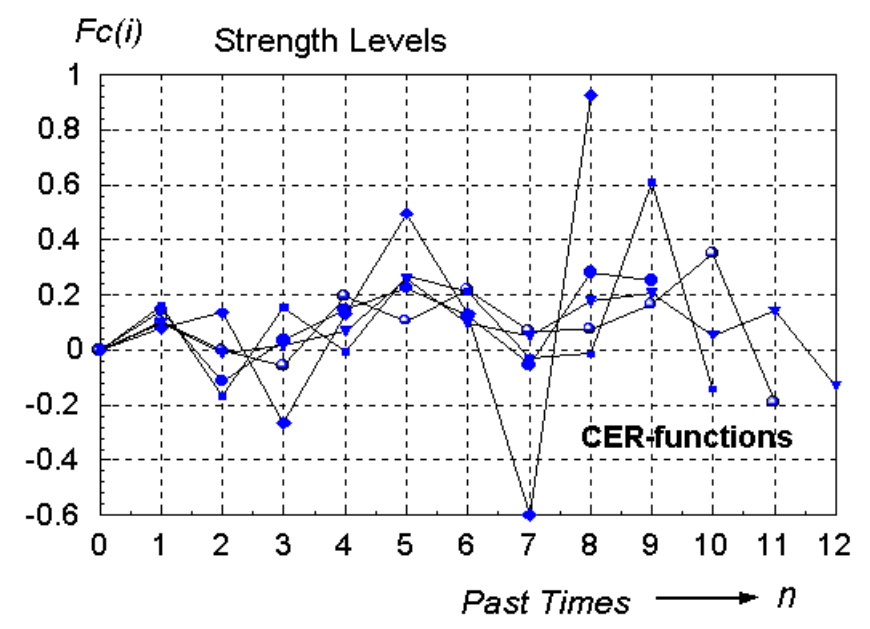

FIG. 19. Identified CER-functions of the Feed-Forward Controllers with Memory Lengths: $n=$ $8,9,10,11$ and 12 .

as a proof of that expectation. It is especially clear from Fig. 21. that a controller with a longer memory length $n_{c}>7$ does not improve the performance significantly, since the older information is no longer related to the current output in this particular simulation.

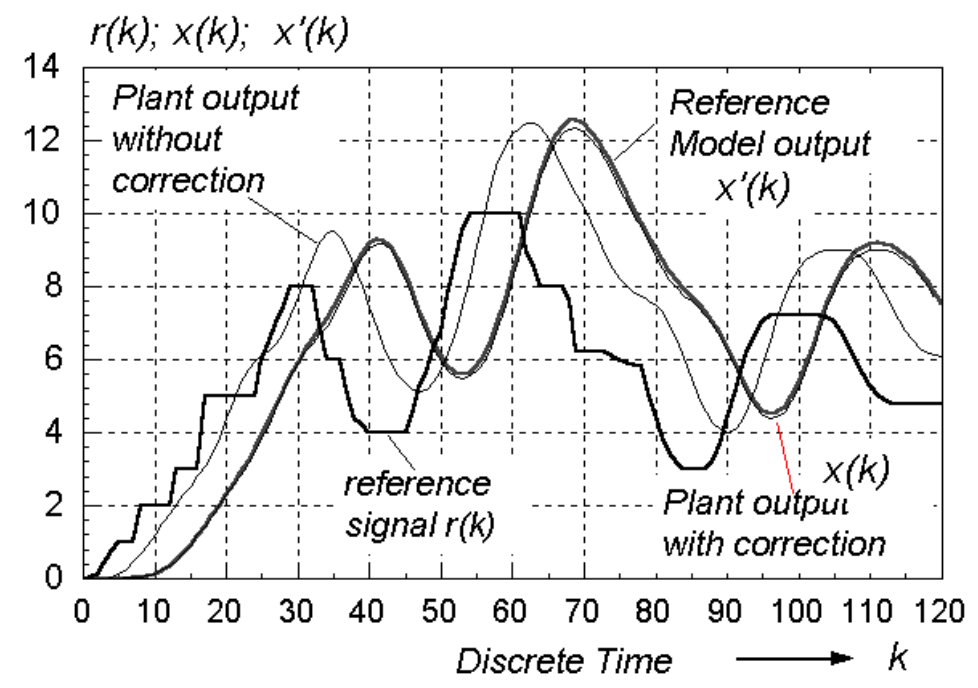

FIG. 20. Performance of the Reference Model Control Based on the Identified Feed-Forward Controller with $n_{c}=7$.

The modified (corrected) signal $u(k)$ that is produced by the feed-forward controller and is further used as an input to the plant, is compared with the original reference 


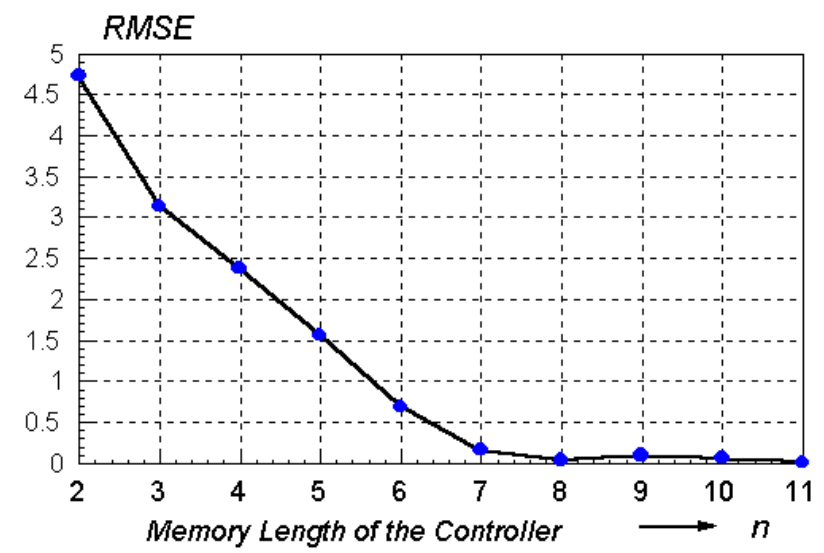

FIG. 21. Rooted Mean Square Error (RMSE) for the Response to the Reference Signal by the Different Controllers.

signal $r(k)$ in Fig. 22.

$r(k), u(k)$

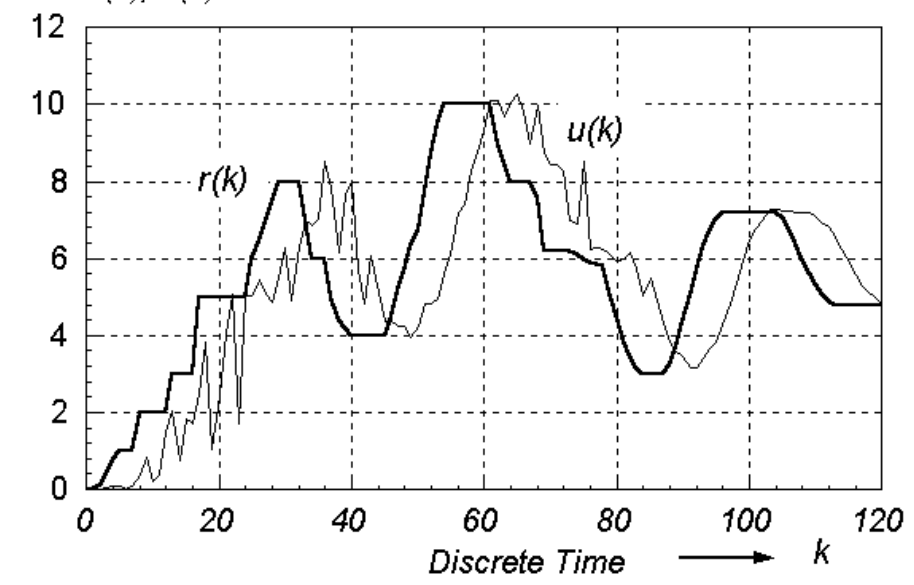

FIG. 22. Comparison of the Reference Signal $r(k)$ with the output $u(k)$ from the Feed-Forward Controller with Memory $n_{c}=7$.

Figure 23. shows the performance of the controllers with shorter memory length: $n_{c}=5,6,7$, based on unit step function reference signal. Obviously, there is an increasing loss of information in the case of shorter memory length that leads to a significant deviation between the performance of the reference model and that one of the serial connection: Controller - Plant.

The above simulations show the importance of the proper choice of the memory length for the reference model controller. 


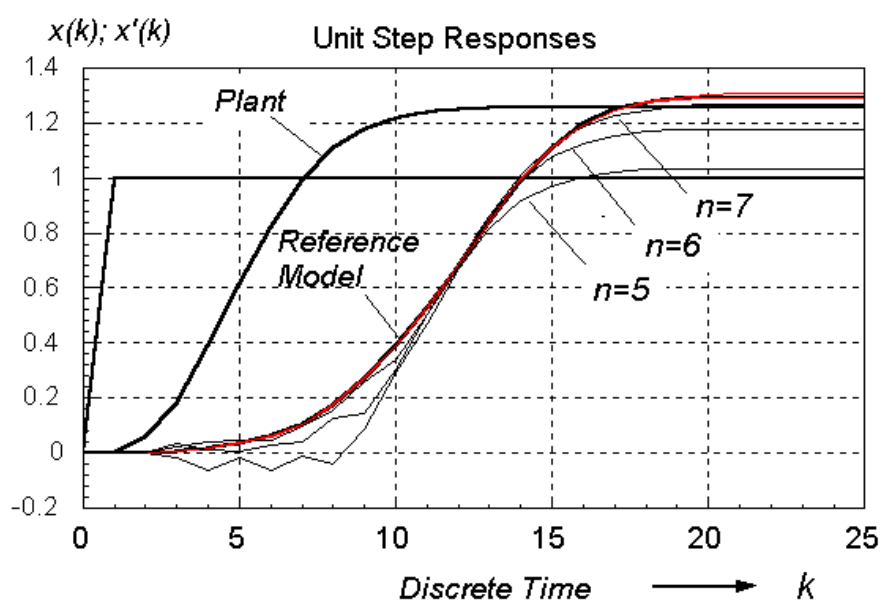

FIG. 23. Performance of the Reference Model Control Based on the Identified Feed-Forward Controller with $n=5,6$ and 7 .

10. Conclusions. An incremental cause-effect relation type of dynamic model (ICER model) is proposed in this paper. It uses the concept of cause-effect relations functions (CER-functions) in order to represent the relationship between the past time changes in control and the change of the current model output. Three identification schemes for this type of model are proposed in the paper. The first one is called Direct Identification and uses the standard Least Mean Squares (LMS) algorithm. The size of this problem is equal to the number of the points $n$ of the CER-functions that is normally high for slow and complex dynamic processes.

Next the Indirect (reduced size) Identification algorithm is presented. It identifies the parameters of a one-dimensional Takagi-Sugeno fuzzy model with a predefined structure that is further used to approximate the cause-effect relation function of the dynamic model. Thus a significant reduction in the size of the identification problem is achieved. Additional advantage of this identification algorithm is that a change in the size nof the real problem does not necessarily mean change of the size of the reduced identification problem. This makes the algorithm computationally efficient with its accuracy depending directly on the predefined structure (the number of the membership functions) of the TS fuzzy model, that is used as a nonlinear approximator.

The third algorithm is called "Soft Guided" Identification that is able to use preliminary human knowledge about the possible or expected type of the plant dynamics. This algorithm is able to produce a more plausible dynamic model especially in the presence of highly noised input-output data. In addition, it gives a lot of flexibility of the identification procedure, being able to make any kind of reasonable balance between "pure data-fitting" and "pure model-type fitting" by changing the weighting coefficient $\lambda$ (or weighting function $\lambda(i)$ ). A further development of this idea includes 
utilizing even a partial knowledge about a specified part of the plant dynamics, which leads to identification of a plausible dynamic model.

Once the dynamic model is identified by use of any one of the proposed identification algorithms, it can be used in various control schemes. In the paper, the identified incremental model is used in several algorithms for predictive control with different horizon lengths and horizon widths. Respective control equations for applying the first control action $u(k)$ have been derived. Finally an original application of the dynamic model to a reference model feed-forward control scheme is presented, analyzed and illustrated.

A variety of simulation results on test examples in the paper illustrate how to use the proposed ICER dynamic model in the above predictive and reference model control strategies. All the simulations are a kind of numerical proof for the applicability of the derived control schemes by use of the ICER model to real plants. Some important directions for future research are the improvement of the model accuracy and control

performance, as well as analysis of the stability and robustness of all the proposed control schemes.

\section{REFERENCES}

[1] C.-H. Lee And C.-C. Teng, Identification and Control of Dynamic Systems Using Recurrent Fuzzy Neural Networks, IEEE Transactions on Fuzzy Systems, 8:4(2000), pp.349-366.

[2] S. Jagannathan, F. Lewis, and O. Pastravanu, Model Reference Adaptive Control of Nonlinear Dynamical Systems Using Multilayer Neural Networks, Proc. of the IEEE Int. Conference on Neural Networks, Vol. 7, 1994, pp. 4766-4771.

[3] B.-S.Chen, C.-S.Tseng, And H.-J. Uang, Mixed $H_{2} / H \infty$ Fuzzy Output Feedback Control Design for Nonlinear Dynamic systems: An LMI Approach, IEEE Transactions on Fuzzy Systems, 8:3(2000), pp. 249-265.

[4] D. Clarke, C. Mohtadi, and P. Tuffs, Generalized Predictive Control-Part 1. The Basic Algorithm, Automatica, 23:2(1987), pp. 137-148.

[5] J. H. Lee And N. R. RickeR, Extended Kalman Filter Based Nonlinear Model Predictive Control, Ind. Eng. Chem. Res., 33(1994), pp. 1530-1541.

[6] J. Abonyi, A. Babushka, M. Setnes, H. Verbrugen, and F. Szeifert, Constraint Parameter Estimation in Fuzzy Modeling, Proc. of the Int. FUZZ-IEEE 1999 Conf., August, 1999, Seoul, Korea, Vol. 2, pp.951-956.

[7] J. Rishalet, Industrial Applications of Model Based Predictive Control, Automatica, 29:5(1993), pp. 1251-1274.

[8] T. Takagi and M. Sugeno, Fuzzy Identification of Systems and Its Applications to Modeling and Control, IEEE Trans. on Systems, Man \& Cybernetics, 15(1985), pp. 116-132.

[9] HaO Ying, General SISO Takagi-Sugeno Fuzzy Systems with Linear Rule Consequent Are Universal Approximators, IEEE Trans. Fuzzy Systems, 6:4(1998), pp. 582-587.

[10] G. Vachkov and T. Fukuda, Simplified Fuzzy Model Based Identification of al Systems, Int. Journal of Fuzzy Systems, 2:4(2000), pp. 229-235.

[11] G. Vachkov and T. Fukuda, Structured Learning of Fuzzy Models for Reduction of Information Dimensionality, Proc. of the $8^{\text {th }}$ Int. Conference FUZZ-IEEE'99, Seoul, Korea, Aug. 22-26, 1999, Vol. 2, pp. 963-968.

[12] G. Vachkov and T. Fukuda, Simplified Predictive Control of Dynamical Systems Based on Incremental Type of Cause-Effect Models, Proc. of the 2001 IEEE International Symposium 
on Computational Intelligence in Robotics and Automation, CIRA-2001, Banff, Canada, 2001, pp. 284-289. 
